\title{
Endoscope captures immigrant tapes in duodenum
}

\author{
Lokesh Locheruvapalli Venkateshappa • Kiran \\ Reddyvari • Avinash Bhat Balekuduru • Umesh Jalihal • \\ Satya Prakash Bonthala Subbaraj
}

Published online: 22 October 2014

(C) Indian Society of Gastroenterology 2014

Taeniasis caused by Taenia solium-the pork tapeworm-or Taenia saginata - the beef tapeworm, and is endemic in Southeast Asia. In a study by Wani et al. [1], the prevalence of T. saginata asiatica was reported to be $7.69 \%$ in rural Kashmir which was secondary to ingestion of measly beef. Most cases of taeniasis are asymptomatic and usually complain of passage of proglottids with stools. However, others present with pruritus ani (77\%), nausea (46\%), abdominal pain (43\%), dizziness $(42 \%)$, increased appetite (30\%), and other mild gastrointestinal symptoms [2]. The diagnosis is generally established by identifying eggs or proglottids in the stool. Single dose of $5-10 \mathrm{mg} / \mathrm{kg}$ of oral praziquantel is the treatment of choice. Taeniasis is rarely detected on endoscopy. We present an incidental endoscopic removal and detection of $T$. saginata asiatica in a case of carcinoma of distal esophagus (Fig. 1). He was asymptomatic and in retrospect he revealed episodic passage of white strings/proglottids in stools and was a beef eater. It is essential to improve the protocol detection of parasitism in meat industries.

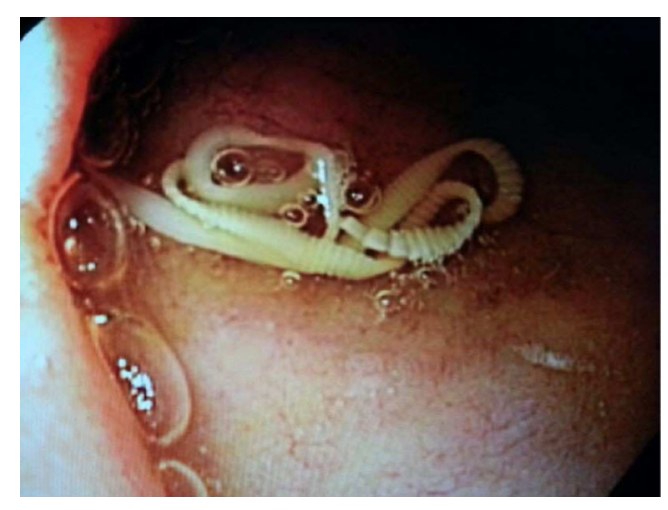

Fig. 1 Endoscopic image of T. saginata asiatica in duodenal bulb

\section{References}

1. Wani S, Ahmad F, Zargar S, Fomda B, Ahmad Z, Ahmad P. Helminthic infestation in children of Kupwara district: a prospective study. Indian J Med Microbiol. 2007;25:398-400.

2. Fan PC, Chung WC, Lin CY, Chan CH. Clinical manifestations of taeniasis in Taiwan aborigines. J Helminthol. 1992;66:118-23.

L. L. Venkateshappa $\cdot$ K. Reddyvari · A. B. Balekuduru •

U. Jalihal $(\bowtie) \cdot$ S. P. B. Subbaraj

Department Gastroenterology, M S Ramaiah Memorial Hospital,

MSRIT Post, Bangalore 560 054, India

e-mail: drumeshj@yahoo.com 